\title{
Non-linear Poincaré analysis of respiratory efforts in sleep apnea
}

\author{
Behbahani $\mathrm{S}^{1}$, Moridani $\mathrm{MK}^{2}$ \\ Department of Electrical Engineering, Garmsar Branch, Islamic Azad University, Garmsar, Iran. \\ sor.behbahani@gmail.com
}

\begin{abstract}
OBJECTIVES: Obstructive sleep apnea (OSA) is a risk factor for hypertension, has effects on cardiovascular system and increases the sympathetic activity. The aim of the study was to evaluate the effectiveness of the non-linear Poincaré plot analysis to predict OSA based on polysomnography (PSG).

METHODS: The database of this study was collected by the sleep laboratory at the Philipps University in Marburg, Germany. It includes 24 PSG of men and women between 27-63 years old with obstructive and mixed sleep apnea. The start and end of apnea events in PSGs were marked. The Poincaré plots of pre-apneic phase including 4-1 minutes before apnea were evaluated. Wilcoxon test was used for statistical analysis.

RESULTS: Poincaré analysis showed that the dynamics of chest and respiratory efforts changed two minutes before the apnea and SD1/SD2 ratios of these parameters significantly increased in the pre-apneic phase $(p \leq$ $0.01)$. The SD1/SD2 ratio of nasal airflow did not show significant difference even in episodes close to apnea. CONCLUSIONS: Our results suggest that Poincaré plot parameters of PSG have the potential to be considered predictors of apnea with the ability to show the dynamic of changes, which could lead to pre-diagnosis or prediction of apnea about 2-3 minutes before its occurrence (Tab. 2, Fig. 4, Ref. 23). Text in PDF www.elis.sk. KEY WORDS: obstructive sleep apnea, Poincaré plot, prediction, PSG.
\end{abstract}

\begin{abstract}
Abbreviations: OSA - Obstructive sleep apnea, PSG - polysomnography, ECG - electrocardiogram, HRV - heart rate variability, SD - standard deviation, CNS - central nervous system, Res - respiratory, Abd - abdominal, Nas - nasal.
\end{abstract}

\section{Introduction}

Sleep is a passive period with elimination of excitation while wakeful state is associated with active excitation of central nervous system (CNS). Previous studies have shown that sleep is independent of wakefulness, generated by a sequence of changes in CNS and is a combination of different stages with clear boundaries. It is an important activity which renews mental and physical health every day and covers one-third of our lives.

Breathing is one of the main body functions and its alterations have been proved to be a risk factor in different patient population (1). The respiratory pump consists of respiratory muscles, structure of the rib cage and abdomen controlled by the brain, spinal cord and the peripheral nerves. Since breathing is established autonomously during sleep, it is affected by many anatomical and physiological parameters, which are different from non-sleep (2). Depending on these situations, various sleep disorders occur. There are lots of well recognized sleeping diseases including dream dis-

${ }^{1}$ Department of Electrical Engineering, Garmsar Branch, Islamic Azad University, Garmsar, Iran, and ${ }^{2}$ Department of Biomedical Engineering, Science and Research Branch, Islamic Azad University, Tehran, Iran

Address for correspondence: Behbahani S, No. 29, Floor 4, Farjam St., Tehran-Pars, Tehran, Iran. Postal Code: 1653989618

Phone: +982177874289, Fax: +982188675452 orders, somnambulism, insomnias, hypersomnias and respiratory sleep disorders. Most of them lead to deterioration of health and decrease quality of life. One of the significant sleep disorders is the respiratory disorder during sleep, which has a higher incidence in adults than in children. It is reported that sudden unexpected deaths during sleep, sleepiness during the day, fatigue and snoring at night are caused by respiratory disorders in sleep. Therefore, regular breathing during sleep is an important activity that plays a vital role in human health (3).

Obstructive sleep apnea, the most common of the different types of sleep-related breathing disorders is partial or complete upper airway obstruction during sleep when air is prevented from entering lungs and covers about $84 \%$ of the cases (4) and is characterized by repetitive cessations of respiratory flow during sleep. Generally, sleep apnea goes undiagnosed as painful symptoms do not appear and patients do not seek medical aid. The most common sleep apnea indicators are daytime sleepiness, irritability, tiredness, low concentration and impaired learning (5). Growing evidence shows that OSA is considered to be a risk factor for hypertension and is independently associated with poor cardiovascular outcomes in general population and has effects on cardiovascular system and increases the sympathetic activity (6-8).

The onset of each OSA is associated with inspiratory efforts against a closed airway, which increase parasympathetic activity leading to a bradycardia, but as the physiological stress builds up during apnea, sympathetic activity predominates. This peaks shortly after the moment of arousal at which there is systemic vasoconstriction, hypertension, and tachycardia present. Termination of OSA requires arousal from a deeper-to-lighter stage of 
sleep or wakefulness (2). This is accompanied by a decrease of blood oxygen over time and mechanical respiratory efforts that are intensified in order to reopen upper airways. If these efforts are not sufficient and the hypercapnia level is dangerous, arousal is generated to reactivate all the peripheral systems and respiration is restored. This episode may repeat hundreds of times in a single night, with serious health implications (9).

The measurement and monitoring of respiration is one of the important functions to check the respiration rate and breathing health. Direct recording of respiratory signals presents practical relevant problems. There are many studies on the topics of detection and quantitative characteristics of sleep disorders, especially sleep apnea using analytical methods from sleep records in the literature. These studies help the physicians during the process of diagnosis and treatment of sleep disorders. The most important research on sleep in the field of biomedical engineering is the measurement and recording of physiological signals during sleep. Detection of sleep apnea from the ECG has been studied by a number of groups during the Computers in Cardiology challenge 2000 (10). One objective of this challenge was to quantify the apnea stress by the number of minutes spent with disordered breathing. The surprisingly good results and methods are summarized in (11).

Polysomnography is a multi-parametric test and the gold standard tool for sleep apnea diagnosis. PSG, which is usually performed at night, is a comprehensive recording of the electrophysiological changes that occur during sleep. The most common signals recorded are electroencephalogram, electromyogram, electrooculogram, electrocardiogram, airflow, and oxygen saturation. The acquisition and analysis of these signals require human experience and specialized equipment (12-14). Moreover, respiratory efforts along with the airflow are used to diagnose patients with sleep-disordered breathing. The effort measurement represents an accurate distinction among physiological and various pathological respiratory patterns. Increasing effort to breathe during a significant reduction or complete suspension of airflow confirms obstructive sleep apnea.

The aim of this study was to assess whether Poincaré plot analysis of PSG signals could yield essential information on the diagnosis or even prediction of OSA. This paper is organized as follows: Section 2 introduces data and method. Section 3 presents the results that are discussed in Section 4. Finally, Section 5 gives the conclusions.

\section{Materials and method}

\section{Database Description}

The database used in this research is free and available on the Physionet website, includes the description of data and the original challenge. This database was used during the annual Computers in Cardiology Challenge in 2000 (10). It consists of 24 GPSs, includes men and women between the ages 27-63 years, with weights ranging from 53-135 kg and apnea/hypopnea index of 10 or more. The PSGs of studied patients exist and include chest and abdominal respiratory efforts, nasal airflow and Spo 2 signals. The start and end of apnea events in PSGs were marked. Data were collected by the sleep laboratory at the Philipps University in Marburg, Germany. The sampling frequency of ECG was $100 \mathrm{~Hz}$.

Apnea scoring was carried out based on standard criteria by experts in sleep scoring with a minute-by-minute resolution. A minute was defined as apnea if at least one apnea or hypo-apnea episode occurs. Otherwise, that minute was defined as normal breathing. This procedure was used for the total sleep time for each subject.

\section{Non-Linear Poincaré analysis}

Poincaré plot is a geometrical representation of a time series in a Cartesian plane to assess and qualitatively visualize a complex non-linear behavior of physiological signals. We defined the data by:

$$
\mathrm{x}=x_{1}, x_{2}, x_{3}, \ldots
$$

The return map will be a plot of the points. $\left(x_{0}, x_{1}\right),\left(x_{1}, x_{2}\right),\left(x_{2}\right.$, $\left.x_{3}\right), \ldots$ A shape of the plot describes the evolution of the system and allows us to visualize the variability of $x_{n}$ as a time series (15).

Researchers fitted an ellipse to the shape of the Poincaré plot and defined two standard descriptors of the plot SD1 and SD2 for quantification of its geometry. These standard descriptors represent the minor axis and the major axis of the ellipse. The description of SD1 and SD2 in terms of linear statistics shows that the standard descriptors guide the visual inspection of the distribution (16).

Descriptors SD1 and SD2 can be defined as:

$$
\begin{aligned}
& \mathrm{SD} 1=\frac{\sqrt{2}}{2} S D\left(x_{n}-x_{n+1}\right) \\
& \mathrm{SD} 2=\sqrt{2 S D\left(x_{n}\right)^{2}-\frac{1}{2} S D\left(x_{n}-x_{n+1}\right)^{2}}
\end{aligned}
$$

In case of heart rate variability (HRV), it reveals a useful visual pattern of the RR interval data by representing both short and long term variations of the signal (17-18). The inherent assumption behind using consecutive RR points is that the "present RR-interval" significantly influences the "following-RR interval".

The Poincaré plots theory can be used to quantify self-similarity in usually periodic functions. Therefore, it can be applied on other dynamics that repeated on specified intervals and time series like respiratory rate. In the normal breathing process, the chest and diaphragm are making efforts to pull air into the lungs; however in apnea the passageway to the lungs is blocked. Monitoring chest wall and abdominal movements to assess the respiratory efforts, as well as airflow through the nose to determine the depth and frequency of breaths could be a useful tool to analyze apnea.

In Poincaré plots analysis, when all intervals of selected time series are plotted, the assumption is made that the points in the central portion of the plot are normal and outlier points are ectopic. It can be interpreted that the points close together appearing in the center of the ellipse show little change, while the scattered points indicate the existence of some kind of disorder or chaos in time series. 
426-432

\section{Statistical analysis}

The results are presented as mean \pm standard deviation (SD). As data have asymmetric distribution, the Wilcoxon test was used to evaluate whether there is any significant change in PSG signals of pre-apneic phase. Statistical analysis was performed using IBM SPSS Statistics version 19 (IBM, Armonk, NY, USA), and differences were considered statistically significant at $\mathrm{p} \leq 0.01$.

\section{Results}

In this research, 24 PSG recordings including men and women between 27-63 years old and 53-135 kg weights were studied. In the first step, the chest respiratory, abdominal respiratory, nasal respiratory and Spo2 rates of each apneic signal were analyzed minute-by-minute for $4 \mathrm{~min}$ before apnea to evaluate whether any significant changes preceded apnea. The duration of apnea and the fluctuations before apnea differ from patient to patient and even differ from event to event in the same patient.

An example of a sleep apnea event is given in Figure 1. In this figure, the signals duration is approximately seven minutes, which include the period of 1 minute before the apnea started in the vertical green line and ended in first red line. The start and end time of the apnea is represented by two vertical red lines. As it can be seen, the chest respiratory, abdominal respiratory, nasal respiratory and $\mathrm{Spo} 2$ rates show some fluctuations in the period prior to apnea. The fluctuation of Spo2 is less than others but in three remain-

Tab. 1. Variations of PSG parameters in pre-apneic phase.

\begin{tabular}{lccc}
\hline Parameters & $4 \mathrm{~min}$ & $3 \mathrm{~min}$ & $2 \mathrm{~min}$ \\
\hline Chest res. effort $(\mu \nu)$ & $-89.81 \pm 120.37$ & $-232.09 \pm 123.90^{*}$ & $-187.59 \pm 333.90$ \\
Abd. res. effort $(\mu v)$ & $-95.41 \pm 176.81$ & $113.9 \pm 327.51$ & $129.2 \pm 508.74$ \\
Nas. Airflow $(L / s)$ & $-66.66 \pm 256.59$ & $-59.04 \pm 207.98$ & $-100.27 \pm 292.67 *$ \\
Spo2 $(\%)$ & $95 \pm 0.29$ & $95 \pm 0.41$ & $94 \pm 1.52$ \\
\hline
\end{tabular}

Res - respiratory, Abd - abdominal; Nas - nasal

*Significance $(\mathrm{p} \leq 0.01)$ of Wilcoxon test
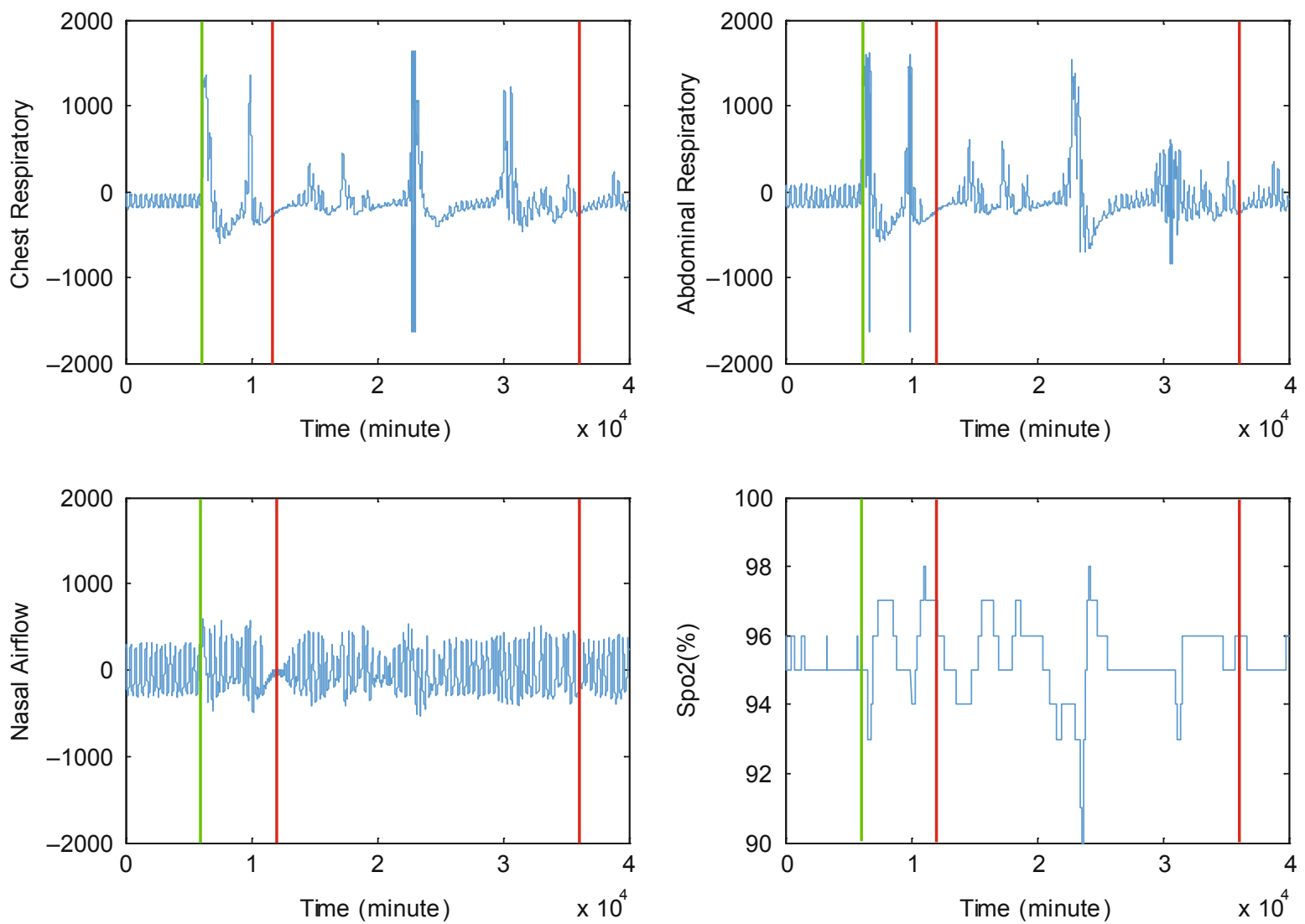

Fig. 1. A sample sleep apnea in one of the studied patients, which includes chest respiratory efforts (top-left), Abdominal respiratory effort (topright), nasal Airflow (bottom-left), SpO2 (bottom-right). The vertical green line and the first red line represent the period of 1 minute before the apnea and the red lines indicate the start and end of apnea. 

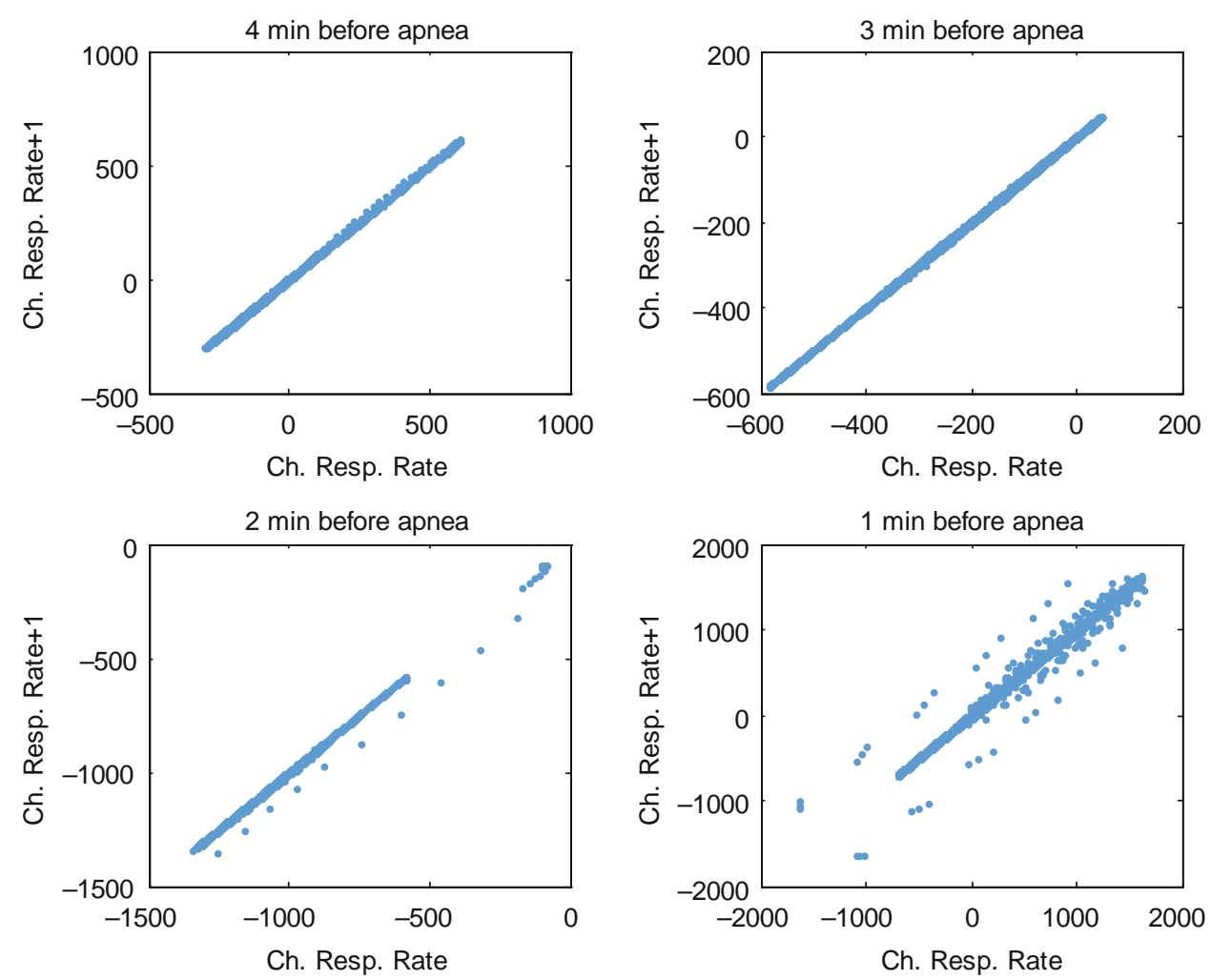

Fig. 2. The Poincaré plots of four episodes of the chest respiratory efforts prior to apnea
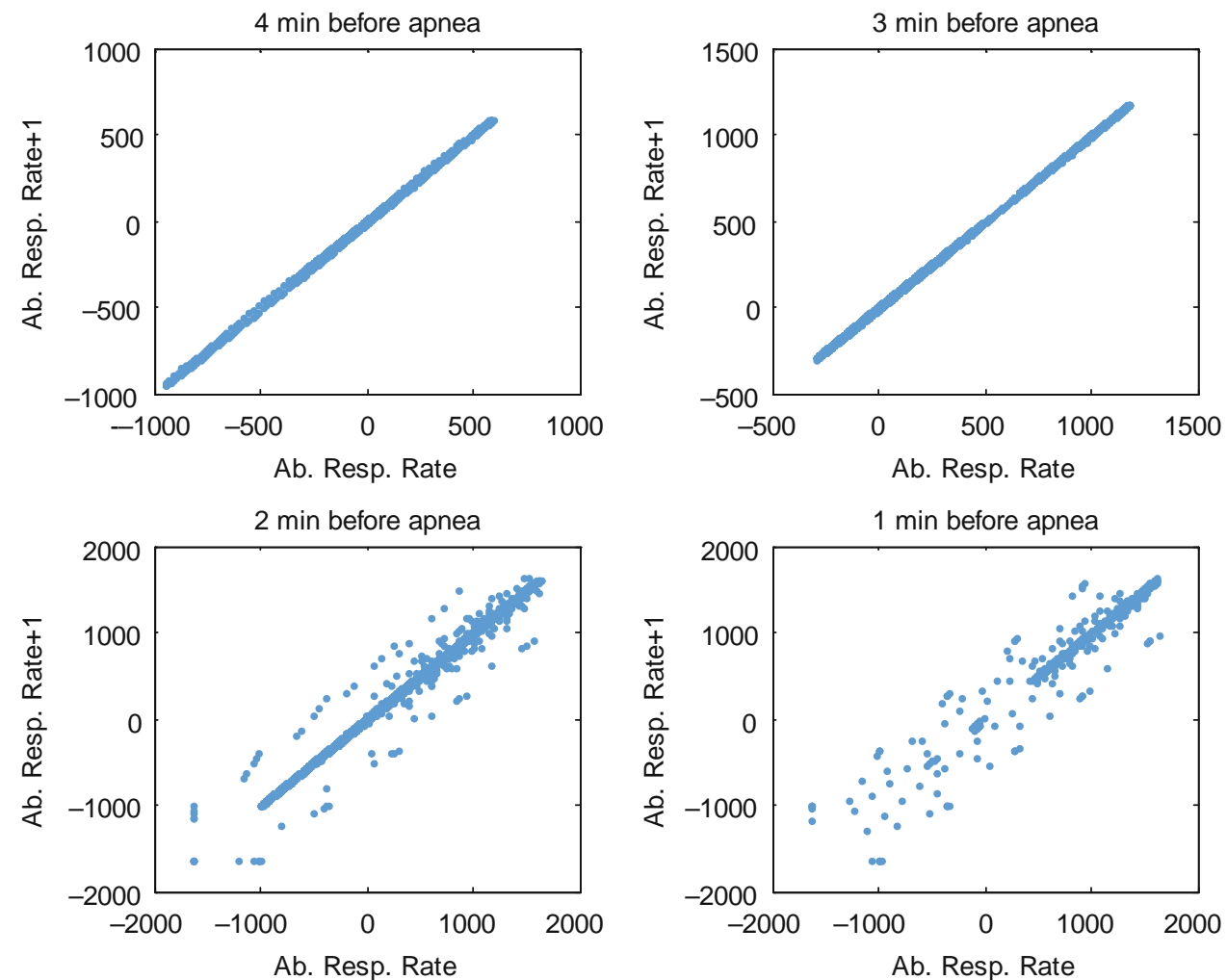

Fig. 3. The Poincaré plots of four episodes of abdominal respiratory rate prior to apnea. 

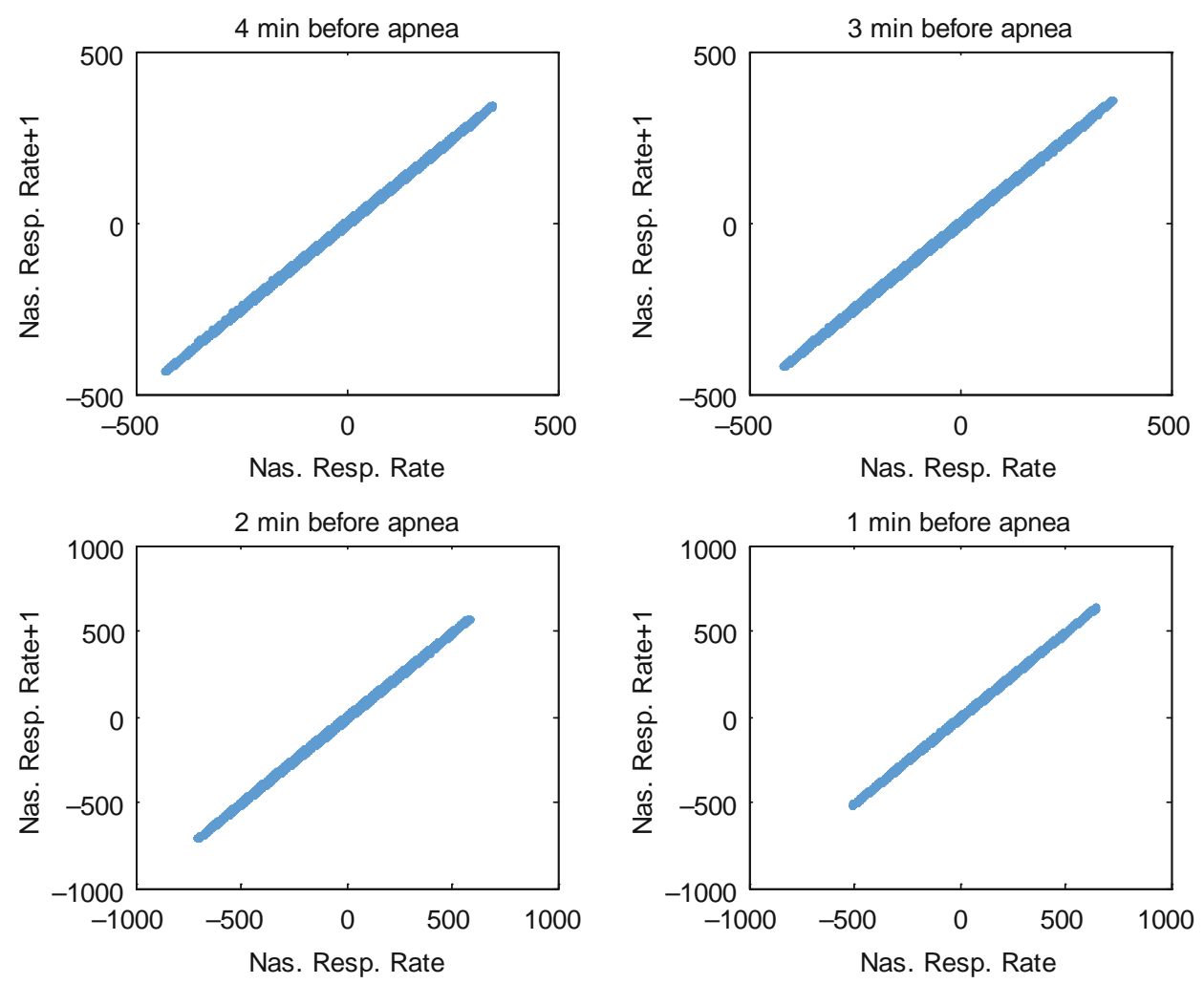

Fig. 4. The Poincaré plots of four episodes of nasal respiratory rate prior to apnea.

ing signals the fluctuations have emerged with greater intensity.

To evaluate the possibility of apnea prediction four minutes before event was considered as the pre-apneic phase, and the mean SD of PSG parameters were measured in this phase. Table 1 represents the variations of PSG parameters before apnea.

Statistical analysis indicates that the chest and abdominal respiratory efforts were significantly higher in periods close to apneic phase. The nasal airflow and Spo 2 showed some amounts of changes in episodes close to apnea; however, the absolute differences were small. As the pre-apneic change in Spo2 was not significant, the Poincare plots were calculated for the remained signals, which are presented in Figs 2-4, respectively.

Figure 2 represents the pre-apneic phase of the chest respiratory efforts in 4-1 minutes before apnea. The Poincaré plots are constructed by plotting each chest respirator effort value against the previous one. An ellipse was fitted to the points in each period. It can be seen that the points of 4-3 minutes before the apnea are close together, and there is no ectopic point in these periods. However, in the episodes of 2-1 minutes before the apnea some ectopic points appear, which emphasize existence of chaotic behavior in chest respiratory effort patterns in episodes close to apnea.

Figure 3 represents the pre-apneic phase of abdominal respiratory efforts in 4-1 minutes before apnea. The Poincaré plots are constructed by plotting each abdominal respiratory effort value against the previous one. It can be seen that similar to the Poincaré plot of the chest respiratory effort, the points in periods of 4-3 minutes before apnea are close together, and there is no ectopic point in these periods. In the episodes of 2-1 minutes before the apnea ectopic points appear. The ectopic points in these two episodes are more than the ectopic points of chest respiratory efforts, which illustrate the different dynamics of chest and abdominal efforts in pre-apneic phase.

In Figure 4, the nasal respiratory effort value was plotted against the previous one. In all episodes, the points are close together, and there is noectopic point in these periods. Table 2 shows Poincare' plot indices of PSG parameters in the pre-apneic phase. In the episodes of 2-1 minutes before apnea, SD1/SD2 ratios of chest and abdominal respiratory efforts were greater than previous

Tab. 2. Poincare' plot indices of PSG parameters in pre-apneic phase.

\begin{tabular}{lccc}
\hline Parameters & $4 \mathrm{~min}$ & $3 \mathrm{~min}$ & $2 \mathrm{~min}$ \\
\hline SD1/SD2 of Chest res. effort & $0.23 \pm 0.04$ & $0.32 \pm 0.09$ & $0.68 \pm 0.17 *$ \\
SD1/SD2 of Abd. res. effort & $0.25 \pm 0.05$ & $0.37 \pm 0.08$ & $0.87 \pm 0.21 *$ \\
SD1/SD2 of Nas. Airflow & $0.22 \pm 0.03$ & $0.24 \pm 0.04$ & $0.91 \pm 0.27 *$ \\
\hline
\end{tabular}

Res - respiratory; Abd - abdominal; Nas - nasal

*Significance $(\mathrm{p} \leq 0.01)$ of a Wilcoxon test 
periods. The SD1/SD2 ratio of nasal airflow did not show significant difference even in episodes close to apnea.

\section{Discussion}

Previous studies for prediction of OSA have used a wide variety of statistical approaches, photographic analysis and clinical and photographic measurements to predict the presence of OSA (19-22). These approaches have been limited by poor sensitivity, specificity, lack of prospective validation and taking too much time for computations and analysis. Moreover, these methods could not represent the dynamic changes of considered parameters such as PSG signals in pre-apneic phase. Despite the ability of non-linear methods and specially Poincaré plots to extract and follow different patterns in complex systems such as HRV and fluctuations of sympathetic and parasympathetic activity, these methods are only included in a few studies related to OSA (23).

The objective of the present study was to evaluate the capability of non-linear Poincaré plots as a predictors of OSA. In this study, we measured the Poincaré indices of PSG signals in the preapneic phase to evaluate whether any significant changes occurs in episodes prior to apnea. Moreover, the statistical changes of PSG parameters were considered to better understand the pattern of fluctuations. Although statistical analysis indicates the fluctuations of PSG parameters in pre-apneic phase, in some patients the pattern of fluctuations and the absolute differences are not significant. It seems that the dynamic changes of PSG parameters could not be identified by statistical or even linear analysis.

The statistical analysis indicates that most fluctuations of PSG signals occurred in one minute before the apnea; however, in some patients these fluctuations were not significant. On the other hand, Poincaré plots of the chest and abdominal respiratory efforts show significant changes from two minutes before the apnea. The pattern of adjacent points in ellipse changed, and the scatter points appeared, which emphasize the chaotic behavior in this phase.

Moreover, increased SD1/SD2 ratios of chest and abdominal respiratory efforts reflect chaotic behavior in these time series. Among the PSG parameters evaluated in this study, abdominal and chest respiratory efforts showed more chaotic behavior and the nasal airflow showed some amounts of chaos due to increase of the SD1/SD2 ratio without representing ectopic points.

The main finding of this study is that patients with OSA have significant changes in dynamics of chest and abdominal respiratory efforts during the pre-apneic phase, which could lead to pre-diagnosis or prediction of the apnea about 2-3 minutes before its occurrence. Therefore, our results indicate that the Poincaré analysis of PSG could be considered as a reliable predictor of sleep apnea.

Further studies will be needed to address some of the limitations of this study. Our study was performed in a small number of patients. A future direction of this work would be to apply our methodology to a larger population including higher number of records with OSA to validate the results. We did not consider the effect of some factors such as gender, age, weight and etc. Moreover, evaluation of HRV along with the PSG could lead to better understanding of the behavior and dynamic changes in pre-apneic phase.
Future work will address the algorithm of OSA prediction considering not only HRV analysis, but also other mentioned factors that can affect the results and should be taken into consideration.

\section{Conclusion}

In summary, we introduced a potential prediction possibility for OSA based on dynamic changes in PSG parameters. Using the non-linear Poincaré analysis we could quantitatively and qualitatively study the OSA and predict apnea $2-3$ minutes before occurrence. The Poincaré plots analysis in pre-apneic phase gives more insight in to the PSG parameters. These changes provide supplementary information about sympathetic and parasympathetic activity of respiratory system under the autonomic nervous system.

\section{References}

1. Correa L, Laciar E, Torres A, Jane R. Performance evaluation of three methods for respiratory signal estimation from the electrocardiogram. In Proceedings of 30th Annual International IEEE EMBS 2008.

2. Shineerson JM. Sleep Medicine: A Guide to Sleep and Its Disorder. Blackwell Publishing 2005; 230-231.

3. Aksahin, M.F. Classification of Sleep Apnea Types, Baskent University PhD Thesis, Ankara, Turkey 2010.

4. Morgenthaler TI, Kagramanov V, Hanak V, Decker PA. Complex sleep apnea syndrome: is it a unique clinical syndrome? Sleep 2006; 29: 1203-1209.

5. White DP. Sleep apnea. Proc Amer Thorac Soc 2006; 3: 124-128.

6. Bradley TD, Floras JS. Obstructive sleep apnoea and its cardiovascular consequences. Lancet 2009; 373 (9657): 82-93.

7. Drager LF, Polotsky VY, Lorenzi-Filho G. Obstructive sleep apnea: an emerging risk factor for atherosclerosis. Chest 2011; 140 (2): 534-542.

8. Marin JM, Carrizo SJ, Vicente E, Agusti AG. Long-term cardiovascular outcomes in men with obstructive sleep apnoea-hypopnoea with or without treatment with continuous positive airway pressure: an observational study. Lancet 2005; 365 (9464): 1046-1053.

9. Guilleminault C, Tilkian A. Dement, The sleep apnea syndromes. Annu Rev Med 1976; 27: 465-484.

10. Computers in Cardiology Challenge 2000. Detecting Sleep Apnea from the ECG. Comp Cardiol 2000; 27: 739-760.

11. Penzel T, McNames J, de Chazal P, Raymond B, Murray A, Moody G. Systematic comparison of different algorithms for apnea detection based on electrocardiogram recordings. Med Biol Engin Comp 2002; 40: 402-407.

12. Beebe DW, Gozal D. Obstructive sleep apnea and prefrontal cortex: Towards a comprehensive model linking nocturnal upper airway obstruction to daytime cognitive and behavioral deficits. J Sleep Res 2002; 11: 1-16.

13. Gottlieb DJ, Vezina RM, Chase C, Lesko SM, Heeren TC, WeeseMayer DE et al. Symptoms of sleep disordered breathing in 5-year-old children are associated with sleepiness and problem behaviors. Pediatrics 2003; 112: 870-877.

14. Chervin RD, Archbold KH, Dillon JE, Panahi P, Pituch KJ, Dahl RE, Guilleminault C. Inattention, hyperactivity and symptoms of sleepdisordered breathing. Pediatrics 2002; 109: 449-456. 
426-432

15. Hoshi RA, Pastre CM, Vanderlei LCM, Godoy MF. Poincaré plots indexes of heart rate variability: relationship with other nonlinear variables. Autonomic Neuroscience, Retrieved July 30, 2013, from Science Direct database on the World Wide Web: http://www.sciencedirect.com. DOI:10.1016/j.autneu.2013.05.004.

16. Liebovitch LS, Scheurle D. Two lessons from fractals and chaos. Complexity 2000; 5: 34-43.

17. Tulppo MP, Makikallio TH, Takala TES, Seppanen T. Quantitative beat-to-beat analysis of heart rate dynamics during exercise. Am J Physiol 1996; 271: H244-H252.

18. Brennan M, Palaniswami M, Kamen P. Do existing measures of Poincaré plot geometry reflect nonlinear features of heart rate variability. IEEE Trans on Biomed Engg 2001; 48: 1342-1347.

19. Sharma SK, Malik V, Vasudev C, Banga A, Mohan A, Handa KK, Mukhopadhyay S. Prediction of obstructive sleep apnea in patients presenting to a tertiary care center. Sleep Breath. Sleep Breath 2006; 10 (3): $147-154$.

20. Kirby SD, Eng P, Danter W, George CF, Francovic T, Ruby RR, Ferguson KA. Neural network prediction of obstructive sleep apnea from clinical criteria. Chest 1999; 116 (2): 409-415.

21. Halpin AP, Bunting JM, Selecky PA, Bosveld SG, Hong D. Nursing Assessment for Predicting Obstructive Sleep Apnea: Community Hospital Approach. Topics Adv Practice Nurs J 2008; 8 (1).

22. Lee RW, Petocz P, Prvan T, Chan AS, Grunstein RR, Cistulli PA. Prediction of obstructive sleep apnea with craniofacial photographic analysis. Sleep 2009; 32 (1): 46-52.

23. Morillo DS, Rojas JL, Crespo LF, Leon A, Gross N. Poincaré analysis of an overnight arterial oxygen saturation signal applied to the diagnosis of sleep apnea hypopnea syndrome. Physiol Meas 2009; 30: 405-420.

Received April 21, 2014. Accepted May 5, 2014. 